\title{
Solving Systems of Linear Equations Based on Approximation Solution Projection Analysis
}

\author{
Jurijs Lavendels* \\ Riga Technical University, Riga, Latvia
}

\begin{abstract}
The paper considers an iterative method for solving systems of linear equations (SLE), which applies multiple displacement of the approximation solution point in the direction of the final solution, simultaneously reducing the entire residual of the system of equations. The method reduces the requirements for the matrix of SLE. The following SLE property is used: the point is located farther from the system solution result compared to the point projection onto the equation. Developing the approach, the main emphasis is made on reduction of requirements towards the matrix of the system of equations, allowing for higher volume of calculations.
\end{abstract}

Keywords - Iterative methods, solution approximation projection, systems of linear equations (SLE).

\section{INTRODUCTION}

The demand for new tools for solving systems of linear equations (SLE) has appeared along with the emerging computing technologies, rapidly developing computation methods of physical process modelling, application of discretization of the calculation area (division into subareas) and substitution of differential operations in approximation to algebraic ones. In compliance with the requirements of finite difference method and finite elements method (FEM) and their modifications, the method for solution of weakly-filled diagonal matrices with the expressed main diagonal with the direct and iterative methods [1], [2] has been elaborated. The methods of effective SLE storage and solution have been elaborated considering the matrix symmetry to the main diagonal both for direct and iterative methods. Over the past years, along with the introduction of new computation techniques (super elements, boundary element method), the need for solving SLE with the help of an ultimately filled matrix, using the matrix without the main diagonal dominance [3], [7] has arisen. Such problem-solving techniques are still being developed, frequently applying iterative methods based on the Gauss-Seidel method [2], [7]. Solving SLE with the help of iterative methods (for instance, the Gauss-Seidel method) supposes correction of one of the unknowns (Fig. 1a) by reducing one certain equation residual; furthermore, other equations are not used in this process [2]. To speed up the convergence of an iterative process, the methods are supplemented with relaxation parameters, which optimise variable speed of the iterative process.
The article explores the approach [4], [5] to solving SLE using the matrix without main diagonal dominance and null main diagonal. The method is rooted in the principle of simultaneous reduction of all SLE residuals in each iteration by changing the values of all the system unknowns (Fig. 1b).

The essence of the solution is an iterative solution of optimization problems, where each iteration consists of two steps:

- the first step - to determine the direction of the successive point location;

- the second step - to determine the optimal successive length of the point displacement.

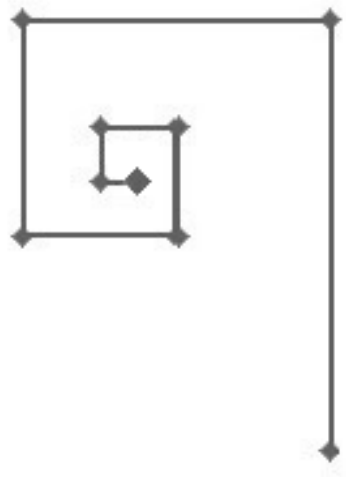

a)

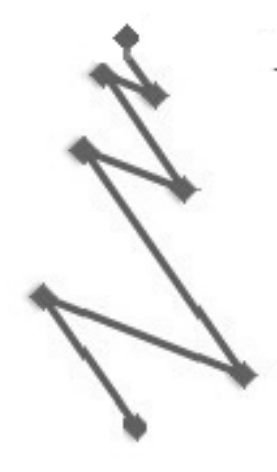

b)
Fig. 1. Correction of one and all unknowns at the iterative solving stage of the equation system.

First, the solution for a two-equation system will be considered, then it will be generalised for any finite dimensional number.

\section{Determination of Point Displacement Direction IN ITERATION}

\section{A. Principles of Determining the Approximation Solution Direction}

It is easy to demonstrate that projections of any point $P(x, y)$ in the $x y$-plane to equations are located closer to the solution of the equation system than the point itself (Fig. 2). The arithmetic average of the projection of all equations is even closer to the solution of the equation system [4], [5].

\footnotetext{
* Corresponding author's e-mail: Jurijs.Lavendelsl@rtu.lv
} 


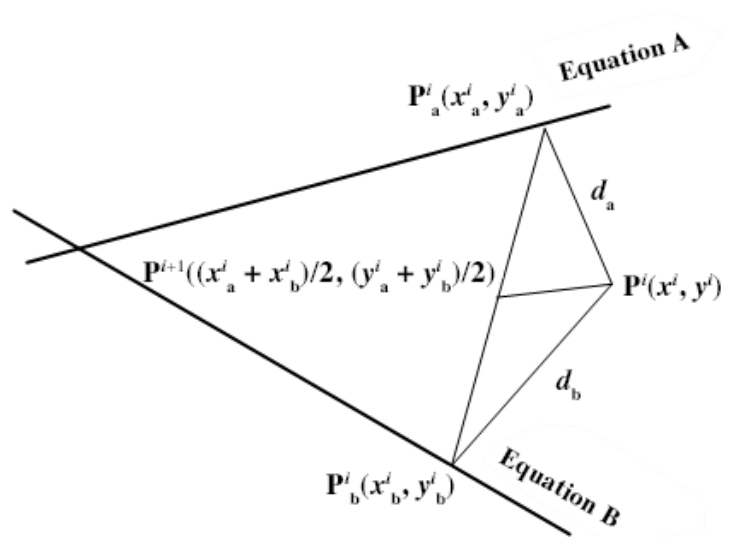

Fig. 2. Determination of the next iterate direction in the iterative system solving.

It follows from the above-mentioned considerations that it is possible to apply the following iterative algorithm to solve the system of equations (Fig. 2):

- the projections of the existing approximation point $\mathrm{P}^{i}(x, y)$ onto equations are determined;

- the midpoint (arithmetic average) $\mathrm{P}^{i}\left(x^{i}, y^{i}\right)$ for projections onto the system equation $\mathrm{A}$ and equation $\mathrm{B}$ is taken as a new approximation $\mathrm{P}^{i+1}\left(x^{i+1}, y^{i+1}\right)$; for equations a and $\mathrm{b}$;

- correction of approximation is repeated iteratively.

\section{B. Direction of Approximation Solution for Larger Equation}

Systems

The considered approach is easily applicable to any finite number of equations.

Therefore, in case of two dimensions, geometrically the equation is a straight line, but a non-contradictory equation system represents two straight lines that intersect, thus forming four angles. In case of three dimensions, one equation geometrically represents a plane, a non-contradictory system represents three planes that intersect. Iterative solving occurs inside the pyramid with three plane derived surface, the apex of the pyramid is sought for. Eight pyramids are formed, whereas the solving process occurs only in one of them.

In case of multiple dimensions, an equation is an $n$-dimension hyperplane, where $n$ is an equation system layer. A non-contradictory equation system is the total of $n$ hyperplanes, which intersect. Iterative solving occurs inside the hyper pyramid, whose hypersurface is formed by $n$ hyperplane equations, the apex of the hyper pyramid is sought for. In case of four and more dimensions, it is impossible to get graphic images of the studied objects, but all mathematical approaches and formulas remain valid. In case of any number of dimensions for the point (iterate) $\mathrm{P}\left(p_{1}, p_{2}, \ldots, p_{n}\right)$, the projection coordinates onto hyperplane

$$
a_{1} x_{1}+a_{2} x_{2}+\ldots+a_{n} x_{n}+b=0
$$

can be calculated as follows [8]. A normal vector to the plane $n=\left(a_{1}, a_{2}, \ldots, a_{n}\right)$ is perpendicular to the plane, so there is a direction vector of the plane that is the nearest distance from point $\mathrm{P}\left(p_{1}, p_{2}, \ldots, p_{n}\right)$ to the plane, i.e., to the point projection onto the plane. Point $\mathrm{P}\left(p_{1}, p_{2}, \ldots, p_{n}\right)$ coordinates are added to the canonical straight line equations:

$$
\left(x_{1}-p_{1}\right) / a_{1}=\left(x_{2}-p_{2}\right) / a_{2}=\ldots=\left(x_{n}-p_{n}\right) / a_{n} .
$$

- straight line equation is expressed in the parametric form:

$$
\begin{aligned}
& \left(x_{1}-p_{1}\right) / a_{1}=t \text { or } x_{1}=a_{1} t+p_{1} ; \\
& \left(x_{2}-p_{2}\right) / a_{2}=t \text { or } x_{2}=a_{2} t+p_{2} ;
\end{aligned}
$$

$$
\left(x_{n}-p_{n}\right) / a_{n}=t \text { or } x_{n}=a_{n} t+p_{n} .
$$

by adding $x_{1}, x_{2}, \ldots, x_{n}$ to the plane equation we acquire:

$$
t=-\left(a_{1} x_{1}+a_{2} x_{2}+\ldots+a_{n} x_{n}\right) / a_{1} a_{1}+a_{2} a_{2}+\ldots a_{n} a_{n} ;
$$

- the coordinates of the projection point are:

$$
\begin{aligned}
& P_{1 \mathrm{proj}}=a_{1} t+p_{1} ; \\
& P_{2 \text { proj }}=a_{2} t+p_{2} ;
\end{aligned}
$$

$$
P_{n \text { proj }}=a_{n} t+p_{n}
$$

In case of any number of dimensions, a new value of approximation coordinates is taken as the arithmetic average of the projections of the previous approximation onto the hyperplanes.

It is not difficult to show that:

- in case of a compatible system, the suggested iterative process approaches the solution with every step;

- the speed of iterative convergence depends on the properties of an equation system, on the angle between the equations in case of two dimensions;

- if equations are contradictory (equations are close to parallels), the process will not converge and the solution will not be found;

- unlike the popular techniques, the approach does not pose strict requirements towards the matrix of equation system - the main diagonal dominance is not important, the main diagonal may have zero entries;

- in each iteration, upon definition of the existing approximation solution projections onto equations and their arithmetic average, the solution direction is determined approximately. The approximation solution direction is close to optimal, but the displacement length is usually significantly lower than the optimal one;

- the technique can be improved. 


\section{Location of a Successive Approximation Solution Point to Equations}

The speed of equation system approximation solution convergence significantly depends on the location of the existing solution point (Fig. 3). Problem solving converges faster if solving occurs in the area of the wide angle (in terms of two-dimensional cases). Still, if SLE is in the angle larger than $90^{\circ}$, iterative process "tries" to leave the area of the wide angle and to move to the adjacent area (Fig. 3).
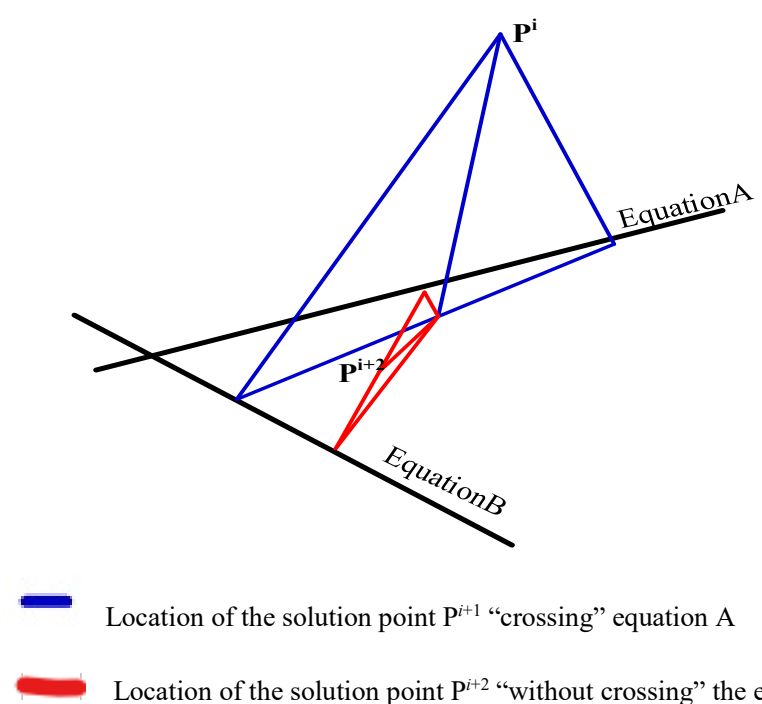

Location of the solution point $\mathrm{P}^{i+1}$ “crossing” equation A

Location of the solution point $\mathrm{P}^{i+2}$ "without crossing" the equations

Fig. 3. Coordinates and solution convergence speed of the successive approximation solution point.

It is important that point $\mathrm{P}^{i}$ is located above, but the successive solution point $\mathrm{P}^{i+1}$ is determined below equation $\mathrm{A}$, according to the coordinates of the calculated point acquired with algorithm (1)-(5). Regardless of whether the successive point and the preceding point are located on one side of the equation or on different sides, each iteration results in the reduction of SLE root mean square error.

Each step of the iteration calculated with algorithm (1)-(5) is directed towards the solution, but is lower than the admissible. The technique of successive iteration step definition is needed for solving the SLE rooted for projections of unknowns to equations.

In cases when the iterative SLE solving process occurs through the equation (Fig. 3), the course of its solution is not interrupted, but it is specified that there is a partially optimal determination of solution direction. It can be demonstrated that the location of the successive point as the arithmetic average of the preceding point projection to equations is not always optimal. It is appropriate to apply the relation

$$
\begin{gathered}
\mathrm{P}^{i+1}(x, y)=\mathrm{P}^{i+1}\left(x_{\mathrm{a}} C_{\mathrm{a}}+x_{\mathrm{b}} C_{\mathrm{b}}, y_{\mathrm{a}} C_{\mathrm{a}}+y_{\mathrm{b}} C_{\mathrm{b}}\right), \\
C_{\mathrm{a}}+C_{\mathrm{b}}=1 .
\end{gathered}
$$

Coefficients $C_{\mathrm{a}}$ and $C_{\mathrm{b}}$ should be determined as the functions of projections $d_{\mathrm{a}}$ and $d_{\mathrm{b}}$ (Fig. 2), defining coordinate of a new point, the largest contribution should be made by the point with the highest value of projection $d_{\mathrm{a}}$ or $d_{\mathrm{b}}$. Evaluation and development of relation (6) effectiveness should consider both achievement and scope of calculations for the computation of the optimal formula (6).

\section{Point Displacement Determination With SuCCESSIVE ITERATION}

\section{A. Equation Solution Residual}

In case of a two linear equation system $F_{1}(\mathrm{x}, \mathrm{y})=0$, $F_{2}(\mathrm{x}, y)=0$, each solution space point is referred to numerical values determined for $F_{1}(x, y)$ and $F_{2}(x, y)$, which will be named as the residual $N_{1}(x, y), N_{2}(x, y)$ (Fig. 4). For equations $F_{1}(x, y)=0$ and $F_{2}(x, y)=0$ the residual value is $N_{1}(x, y)=0$ and $N_{2}(x, y)=0$, respectively. On the one side, the equation has a negative residual value, on another side - positive. On the selected straight line, the residual changes linearly within the solution space.

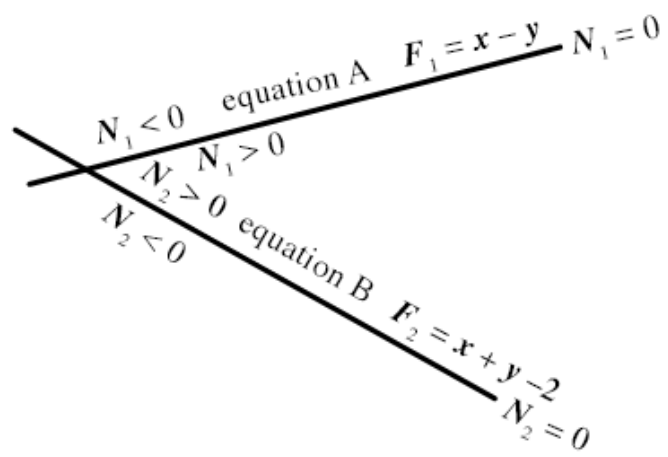

Fig. 4. Residual $N_{1}$ and $N_{2}$ value operators in the sectors of the calculated area.

Equation residuals with the known direction vector can be applied to solving SLE. The solving algorithm should include determining the approximation solution of the specified precision:

- determining the successive approximation point (direction vector) by applying $(1,2,3,4,5)$;

- moving in the specified vector direction at a defined step, while at least one of the system residuals changes its operator.

The further solution can be refined, for instance, using dichotomy principles. Introduction of the approach into practice is going slowly, as the entire residual of equations must be considered at each step.

\section{B. The Equation Squared Residual and the SLE Squared Residual}

Application of the residual can be supplemented with the squared residual of the equation, which is defined as the square of equation residual. The equation squared residual (ESR) on the selected line changes non-linearly, it is a squared function and has only non-negative values.

The squared residual can be applied in the same way as a common residual, by replacing the finding of the operator changes with the finding of the minimal values of the squared residual. ESR characterises the distance from each solution space point to the equation (more precisely, the square of the 
distance). The SLE squared residual (SLESR) is defined as the sum of all squared residuals of equations in the system, as is in case of two equations:

$$
N_{k}(x, y)=N_{1}(x, y)^{2}+N_{2}(x, y)^{2} .
$$

The system squared residual (SSR) offers substantially new properties; this is a smooth non-negative function dependent on all SLE equations, which characterises all processes related to all equations in the system.

In each solution space point $\mathrm{P}(x, y)$, SLESR characterised the distance from a solution space point to the SLE solution as a scalar value. The point of the SLE solution is $N_{k}(x, y)=0$. On the straight line that passes by the SLE solution point, SLESR changes quadratically and achieves the minimum (not null) at the projection of the solution point onto the straight line (Fig. 5). It is possible to determine individual equation squared residuals on the straight line (Fig. 5). ESR value is null (achieves the minimum) at the point, where the straight line crosses the equation. SLESR and ESR minimums on the straight line are mutually displaced (Fig. 5)

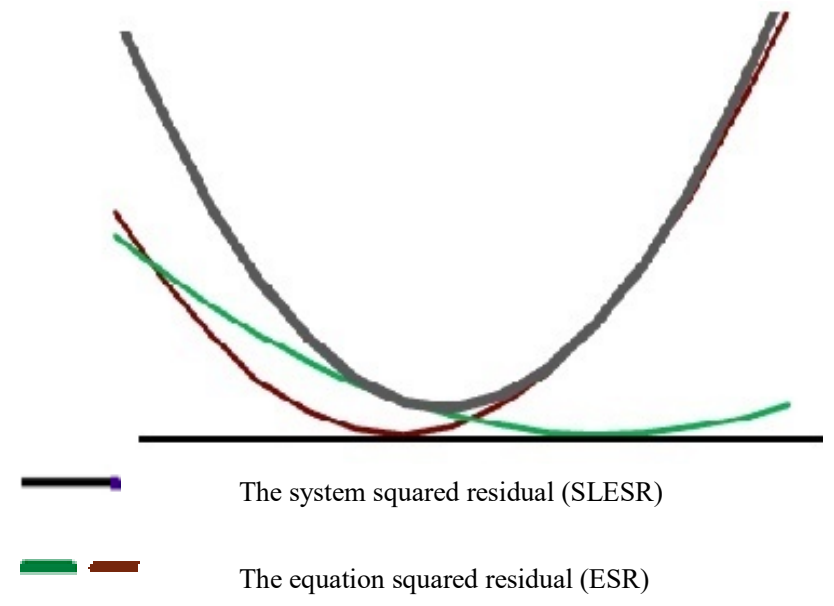

Fig. 5. SLESR and ESR on the straight line in the vicinity of the SLE solution.
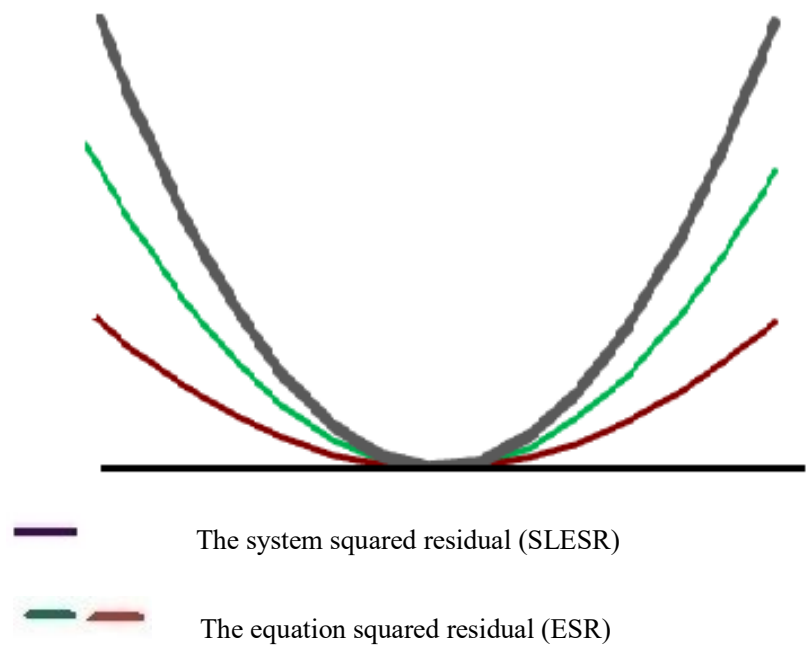

Fig. 6. SLESR and ESR on the straight line going through the SLE solution point.
On the straight line that passes by the SLE solution point, SLESR changes quadratically and achieves the minimum (null) at the solution point (Fig. 6). It is also possible to determine individual equation squared residuals on the straight line (Fig. 6). ESR value is null (achieves the minimum) at the SLE solution point. SLESR and ESR minimum values on the line are not mutually displaced (Fig. 6). Applying the description of SLE properties given above, the SLE solution task can be formulated as follows.

SLE solving is an optimization task - to find the solution space point of the given SLE, where SLESR is 0.

The solving process represents accomplishment of iterative operations:

- $\quad$ to determine the direction vector approximation to SLE solution point using the successive solution space point technique in the rooted approximation solution projection analysis (1)-(5);

- $\quad$ to find the next SLE approximation solution using SLESR extrapolation (Fig. 7).

\section{Calculation of the Successive Approximation Solution Using SLESR Extrapolation}

If the direction of the existing approximation displacement is determined, the successive displacement step can be defined as follows (Fig. 7):

- select three points in the certain displacement direction, for instance, $\mathrm{a}, \mathrm{b}$, c with the step size, one length, two lengths, three lengths, the length is determined by the $(1-5)$ technique;

- SLESR values $N_{k}(\mathrm{a}), N_{k}(\mathrm{~b}), N_{k}(\mathrm{c})$ should be determined at three points;

- build a parabola in these points using the coordinates and SLESR values of the selected points;

- in the parabola minimum point coordinates (function $N_{k}$ derivative is equal to null), take the next approximation solution $x$.

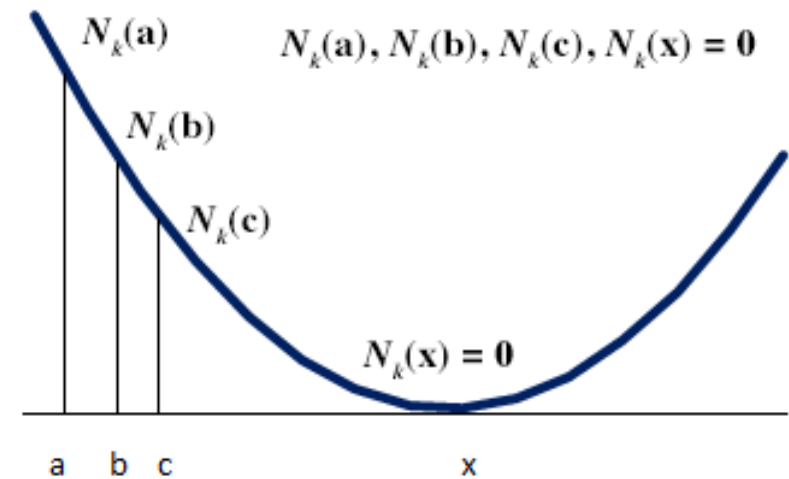

Fig. 7. Successive iteration in determination of a displacement step size.

The process is repeated until the above specified SLESR value is achieved. 


\section{APPROBATION}

The described technique has been approbated in the demo prototype designed for up to 4-equation SLE solving:

- using the technique rooted in the approximation solution projections analysis (1)-(5);

- using the technique rooted in the approximation solution projections analysis (1)-(5) supplemented with SLESR extrapolation.

For instance, the following equation system was solved:

$$
\left[\begin{array}{rrrr}
-6 & 8 & 1 & 1 \\
1 & 4 & -1 & -1 \\
1 & 5 & 0 & 7 \\
-1 & -1 & 2 & 2
\end{array}\right] \cdot\left\{\begin{array}{l}
x \\
y \\
z \\
v
\end{array}\right\}+\left\{\begin{array}{l}
3400 \\
4600 \\
7800 \\
4000
\end{array}\right\}=\left\{\begin{array}{l}
0 \\
0 \\
0 \\
0
\end{array}\right\}
$$

Using the technique rooted in the approximation solution projections analysis (1)-(5), the given system was solved through 80 iterations and the correct solution of the system was found $\{200,400,600,800\}$ with the accuracy of 6 significant figures (Fig. 8).

The solution analysis demonstrates:

- that if the solution is based on the equation (Fig. 8), the behaviour of the solution convergence changes in certain iterations, for example, iteration 33;

- in the beginning, solving can pass through the equation multiple times (Fig. 8);

- using the technique rooted in the approximation solution projections analysis (1)-(5) and in case of SLESR extrapolation, the path of solving is "broken", which is due to squared SLESR extrapolation.

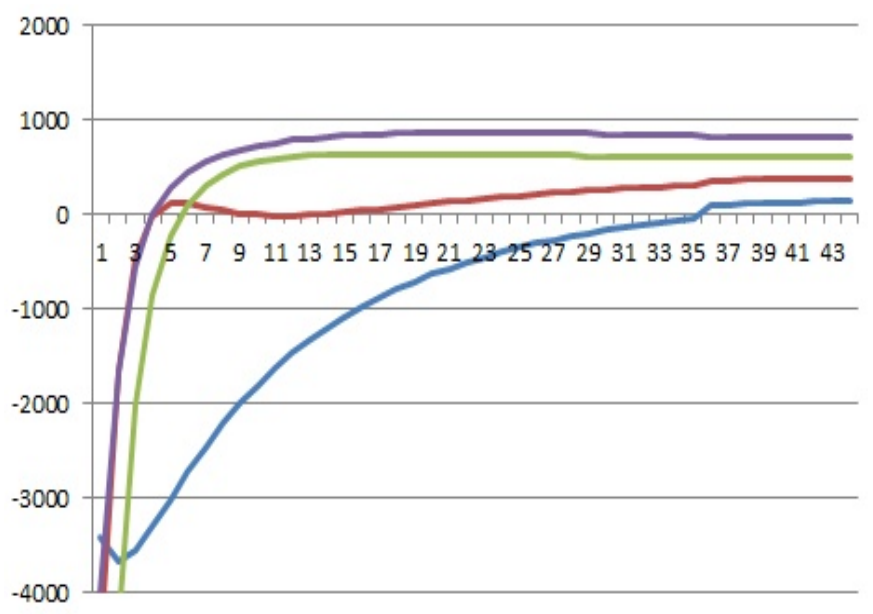

Fig. 8. Solution convergence by the technique rooted in the approximation solution projection analysis (1)-(5).

Using the technique rooted in the approximation solution projections analysis (1)-(5) supplemented with SLESR extrapolation, the solving process required 4 times fewer iterations (Fig. 9). It was impossible to compare the efficiency of the solution with the classical techniques, as due to bad matrix it was possible to solve the system only with the given techniques.

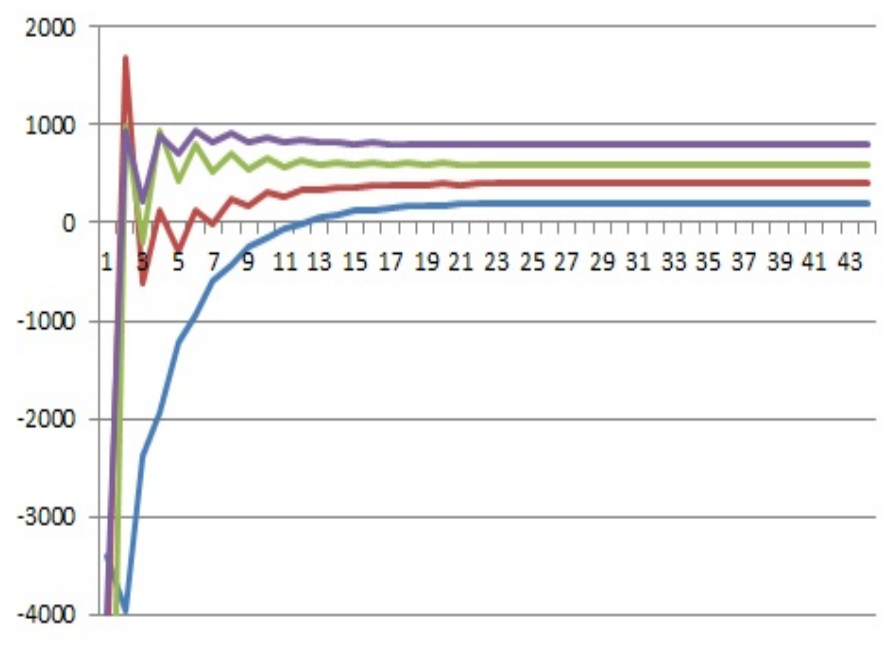

Fig. 9. The solution convergence using the technique rooted in the approximation solution projections analysis (1)-(5) and SLESR extrapolation.

Here, using iterations, all four system unknown adjustments can be understood; furthermore, in order to determine the solution step size, algorithm (1)-(5) was used to define the direction of correction and three ESR calculations in order to find the correction value.

The results of both techniques show that at the beginning of the iterative process both techniques rapidly converge, upon achieving the certain solution value, the speed of convergence decreases and in the end approaches asymptotically the SLE solution. It is evident that applying the technique rooted in the approximation solution projections analysis, solution convergence occurs slower than in case of application of both the technique rooted in approximation solution projections analysis (1)-(5) and SLESR extrapolation, although the solving process is "smoother".

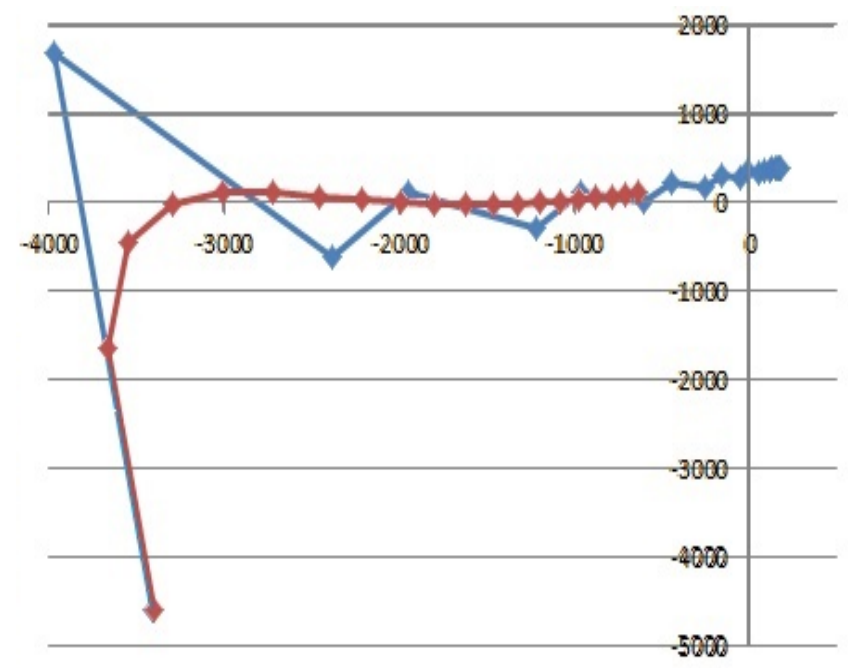

the technique rooted in the approximation solution projections analysis (1) $-(5)$

the technique rooted in the approximation solution projections analysis (1)-(5) and SLESR extrapolation

Fig. 10. 20 first iterations of the solution process, convergence in the projection onto the $x y$ plane. 
The detailed analysis of the solving technique is given in Fig. 10. It is evident that using the technique rooted in the approximation solution projections analysis (1)-(5) and SLESR extrapolation, the process of convergence occurs essentially faster, it is obvious that with the same number of iterations the process has approached the solution much closer. Furthermore, the "broken" profile of the iterative process (snake line) does not affect the quality of the solution. It appeared to be impossible to evaluate quantitatively "the snake line" effect on the number of necessary iterations using the introduced analytical tools. In the beginning, the "passing" of the solution through the equation does not impact much the speed of convergence. At the final stage of the iterative process of solving, passing across the equation reduces the solving step size. This is why the final stage of solving needs additional research, concerning the development of formula (6) and feasibility.

Solving multiple equations shows that SLE matrix also affects the time of calculations.

\section{CONCLUSION}

SLE solving technique rooted in the approximation projections to equations analysis practically functions, posing substantially fewer requirements towards the SLE matrix. Implementation of a certain kind of optimization algorithm lies at the essence of the solution. Introduction of the technique does not pose strict requirements towards system matrix storage.

The first steps in the use of SLE solving technique rooted in the approximation solution projection analysis and determination of the successive iteration step size have been made. Implementation of the techniques into the demo prototype has been accomplished, which confirms the effectiveness of the conducted research. Enhancements to the technique for practical engineering purposes need further research.

The results of the conducted research demonstrate:

- the technique needs substantially more arithmetic operations than the widely used algorithms, although it is applicable to the SLE remaining beyond the capacities of the widely used algorithms;

- the technique has been introduced as an optimization tool;

- the technique does not need complex algorithms to store the SLE matrix;

- the first steps in research of the techniques rooted in the approximation solution projections analysis have been made.

\section{REFERENCES}

[1] B. Demidovich I. and Maron, The Basics of Numerical Methods, (in Russian). Moscow: Nauka, 1970.

[2] I. Bronschtein and K. Semendjajev, Mathematical Manual for Engineers and Students, (in Russian). Moscow: Nauka, 1981.

[3] A. Tsybenko, A. Konyukhov, and H. Tsybenko, "Numerical Method for Determining Stiffness Characteristics of an Arbitrary Form Superelement," Applied Computer Systems, vol. 18, pp. 52-56, Dec. 2015. https://doi.org/10.1515/acss-2015-0019

[4] J. Lavendels, "Another Approach for Solving a System of Linear Equations," Boundary Field Problems and Computer Simulation, vol. 56, pp. 38-41, 2017. https://doi.org/10.7250/bfpcs.2017.005

[5] M. Kryshchuk and J. Lavendels, "Iterative Method for Solving a System of Linear Equations," Procedia Computer Science, vol. 104, pp. 133-137, 2017. https://doi.org/10.1016/j.procs.2017.01.085

[6] A. Heck A., Introduction to MAPLE. New-York: Springer-Verlag, Inc., 1996. https://doi.org/10.1007/978-1-4684-0484-5

[7] M. Trott, The Mathematica GuideBook for Numerics. New-York: Springer-Verlag, Inc., 2006. https://doi.org/10.1007/0-387-28814-7

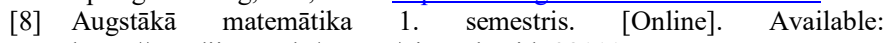
https://estudijas.rtu.lv/course/view.php?id=38111

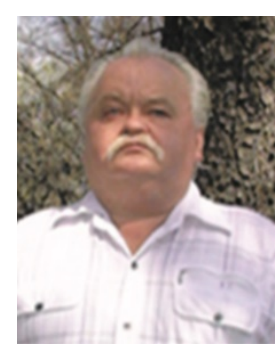

Jurijs Lavendels, Dr. sc. ing., Professor.

His current research interests include computation methods with discretization and algebraization for design and modelling physical processes. E-mail: Jurijs.Lavendelsl@rtu.lv ORCID iD: https://orcid.org/0000-0002-8448-4705 